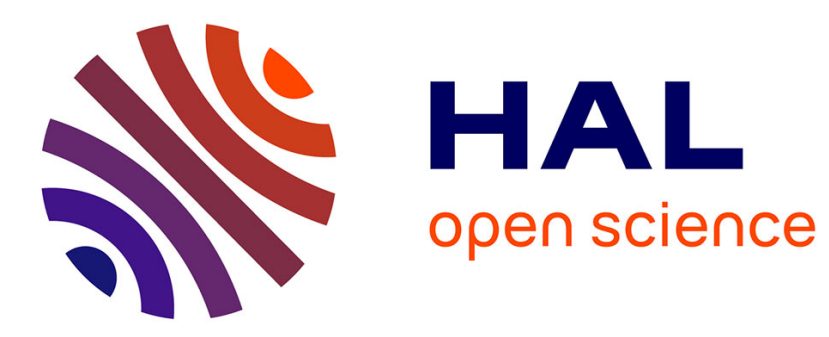

\title{
Whole-Body Manipulation
}

Olivier Stasse, Ludovic Righetti

\section{To cite this version:}

Olivier Stasse, Ludovic Righetti. Whole-Body Manipulation. Encyclopedia of Robotics, Springer Berlin Heidelberg, pp.1-9, 2020, 978-3-642-41610-1. 10.1007/978-3-642-41610-1_187-1 . hal03045448

\section{HAL Id: hal-03045448 https://hal.science/hal-03045448}

Submitted on 8 Dec 2020

HAL is a multi-disciplinary open access archive for the deposit and dissemination of scientific research documents, whether they are published or not. The documents may come from teaching and research institutions in France or abroad, or from public or private research centers.
L'archive ouverte pluridisciplinaire HAL, est destinée au dépôt et à la diffusion de documents scientifiques de niveau recherche, publiés ou non, émanant des établissements d'enseignement et de recherche français ou étrangers, des laboratoires publics ou privés. 


\section{Whole-Body Manipulation}

Olivier Stasse and Ludovic Righetti

Synonyms: Loco-manipulation

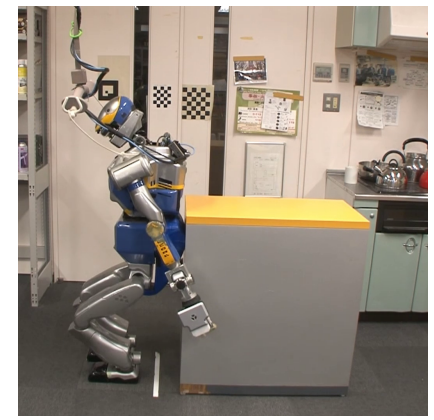

Fig. 1: Example of whole-body manipulation from Murooka et al. (2017): The humanoid robot HRP-2 is trying to push a heavy furniture with his back. HRP-2 has several contacts: surface to surface contacts with the ground and with the object to manipulate. It has also contacts with the object and the end-effectors. The difficulty is to keep the robot's balance while providing enough force to move the object.

Olivier Stasse

LAAS-CNRS, Gepetto TEAM, Toulouse, France e-mail: ostasse@laas.fr

Ludovic Righetti

Tandon School of Engineering, New York University, Brooklyn, USA e-mail: ludovic.righetti@nyu.edu 


\section{Definition}

Whole-body manipulation refers to the manipulation of objects that necessitate the use of all or most of the degrees of freedom of a robot beyond the use of its arms. This term usually applies to robots with kinematic structures that are more complicated than a fixed-base manipulator, for example humanoid robots. Robots capable of whole-body manipulation are generally not grounded to the floor and very redundant. A humanoid robot moving a cabinet is a prime example where movement of the entire body is necessary to manipulate the object.

\section{Overview}

Whole-body manipulation offers opportunities distinct from traditional manipulation settings, yet raising significant challenges. To underline the field specificity, consider a robot moving a heavy cabinet by pushing it with his back as shown in Figure 1. This action necessitates the movement of the entire body to, at the same time, keep balance and carry the object to a desired location. Robots capable of wholebody manipulation are characterized by 1) their high redundancy, often constituted of several kinematic chains (e.g. a humanoid robot with two arms, two legs and a torso), 2) their floating base which means that they are not fixed to the ground but can move in the environment, for example using legs or wheels and, 3) their ability to create multiple contacts with the environment and objects using various parts of their body. ${ }^{1}$ These features significantly increase the accessible workspace and size of objects that can be manipulated, thus enhancing the versatility of the robots and unlocking novel applications in unstructured and complex environments.

The manipulation of sizable objects that require the movement of the entire body has a direct impact on the ability of the robot to maintain balance. It is therefore not possible to decouple balance and locomotion behaviors from the manipulation task anymore. This renders the problem particularly challenging in every aspect of the system design, from the conception of hardware to high-level planning algorithms. The robot needs sufficient strength in all parts of the body, in particular in its arms (when manipulating heavy object, or performing complex locomotion), while retaining a small form factor and weight. It also necessitates multi-modal sensor modalities and estimation algorithms able to evaluate balance quality and distinguish between interaction forces produced by the manipulated object and the ones coming from external disturbances. Control algorithms need to concurrently regulate balancing, locomotion and manipulation behaviors exploiting all the available redundancy. The large number of degrees of freedom and necessity to satisfy dynamic constraints further challenge the design of efficient motion planning algorithms.

${ }^{1}$ The three features are not strictly necessary but rather commonly found on robots used to perform whole-body motion. 


\section{Key Research Findings}

\section{Mechatronics}

To enable whole-body manipulation, robots need to have a sufficient number of degree of freedoms. To manipulate a box in 6 directions a robot needs at least 6 degrees of freedom. In order to make more complex motions such as pivoting, sliding or pushing heavy objects, the robot needs to have a large range of possible motions and cannot remain fixed to the ground. This calls for free-floating redundant kinematic structures including wheeled or legged robots.

To perform successful whole-body manipulation, it is important to regulate interactions forces, including internal forces due to closed kinematic chains resulting from multiple contacts. Ideally one would like to directly control joint torques or forces to enable safe interactions. The TORO robot presented in Englsberger et al. (2014) is working in torque control mode thanks to an embedded torque sensor, a $3 \mathrm{KHz}$ internal low-level control loop (Albu-Schäffer et al. (2007)) and an actuator design enabling high bandwidth torque control. Walking is still quite challenging for torque-controlled bipedal robots, with the recent exception of the work presented in Mesesan et al. (2019). However several quadrupeds using torque control are already successfully walking and performing whole-body motions, see Pavlichenko et al. (2018) for an example with a centaur like robot. Position control is another successful control scheme, especially since many robots do not offer torque control capabilities. In this case, external contact forces are taken into account using a 6D force sensor at the end of each limb and implementing an admittance control scheme, such as in Murooka et al. (2015). Stasse and Flayols (2019) give a detailed overview of the requirements for humanoid robots to accomplish tasks such as whole-body manipulation.

\section{Perception}

Perception is fundamental to understand the states of the robot, the object to be manipulated and the environment. As mentioned previously, internal force perception can be provided by proprioceptive actuators through current measurements (Wensing et al. (2017)), force/torque sensors, and/or modeling of the actuators to enable the reconstruction of joint torques (Nori et al. (2018)). The interaction between the robot and the environment is typically perceived using $6 \mathrm{D}$ force sensors at the end effectors of the robot. They can be used, for example, to identify the object's dynamical parameters and control contact forces (Hasegawa et al. (2018)). Sensors such an Inertial Measurement Unit are desirable to perceive angular velocities and linear accelerations. They are mandatory to perceive the gravity field and constitute a central sensory modality to maintain balance. 
Before contacts are made, vision is used to estimate the object state. Classical approaches are trying to find the pose which matches the current image, but more recent findings are using Deep Learning to provide an estimate of the object pose (Kendall et al. (2015)). Recent contributions aim to further fuse visual and force information to improve contact detection and prediction, as in Lee et al. (2019). 2D (Rioux and Suleiman (2018)) or 3D SLAM is also an important feature to navigate and perform whole-body manipulation

Tactile and proximity sensor are providing important information: the location of external forces applied to the robot. They are for instance necessary when multiple contacts occur between the robot and the object. This is helpful to maintain the surface of an object in contact with the surface contact when doing whole-body manipulation (Mittendorfer et al. (2015)).

Ideally, all these sensing modalities would be fused to provide various estimates of the robot, object and environment and their physical interactions. However, a comprehensive integration of multi-modal sensing remains an open problem both from an algorithmic and a hardware point of view. Robots typically carry few sensors when compared to their animal counterparts and the information carried in these sensors is not yet fully exploited.

\section{Centroidal dynamics}

It is important to relate the effects of object manipulation to the balance of the robot for two reasons: 1) to ensure that object manipulation does not affect the ability of the robot to maintain balance and 2) to ensure that supporting contacts can facilitate manipulation, e.g. pushing on legs to lift a box or taking a step to move a shelf forward. It is therefore crucial to understand how forces created at multiple contacts points throughout the robot's body can concurrently ensure balance and object manipulation objectives. The fundamental relation between external contact forces and overall robot motion is captured by the centroidal dynamics, expressed in an inertial frame located at the robot's center of mass (Orin et al. (2013))

$$
\left[\begin{array}{c}
\mathbf{J}_{\mathrm{CoM}} \dot{\mathbf{q}} \\
\mathbf{A}(\mathbf{q}) \ddot{\mathbf{q}}+\dot{\mathbf{A}} \dot{\mathbf{q}}
\end{array}\right]=\left[\begin{array}{c}
\dot{\mathbf{x}}_{\mathrm{CoM}} \\
\dot{\mathbf{i}} \\
\dot{\mathbf{k}}
\end{array}\right]=\left[\begin{array}{c}
\frac{1}{m} \mathbf{l} \\
m \mathbf{g}+\sum_{i} \mathbf{F}_{i} \\
\sum_{i}\left(\mathbf{x}_{i}-\mathbf{x}_{\mathrm{CoM}}\right) \times \mathbf{F}_{i}
\end{array}\right]
$$

where $m$ is the robot mass, $\mathbf{x}_{\mathrm{CoM}}$ the center of mass position, $\mathbf{l}$ and $\mathbf{k}$ the overall linear and angular momentum of the robot expressed at its center of mass and $\mathbf{F}_{i}$ the external contact forces applied at the contact point $\mathbf{x}_{i} \cdot \mathbf{q} \in \mathbb{R}^{n} \times S E(3)$ are the generalized coordinates of the robot describing the position of its $n$ joints and its position and orientation in space. The centroidal momentum matrix, A, maps velocities into linear and angular momentum. Additionally, a model needs to be provided which will add additional constraints to the equation. For example, each contact force can be constrained to stay in a second-order friction cone. 
These equations link the overall motion of the robot to all external contact forces arising from manipulation and locomotion behaviors. They are typically used as constraints in whole-body manipulation control and planning algorithms to ensure consistency between contact forces and robot motion. The constraints raise several challenges, as they define non-linear and non-convex surfaces. Sampling combinations of compatible contact forces and robot configurations can be quite inefficient for motion planning and more importantly connecting various combinations is challenging as it necessitates the resolution of non-convex optimal control problems. Furthermore, while the linear momentum dynamics can be integrated into a well defined quantity, i.e. the robot center of mass, the angular momentum dynamics is not integrable into a well defined physical quantity. Indeed, the inertia of the system is constantly changing as the robot moves. The angular momentum can in this regard be thought as a non-holonomic constraint (Wieber (2006a)).

While linear approximations of this dynamics, such as the linear inverted pendulum model (Kajita et al. (2001)), have been successfully proposed for legged locomotion they remain very limited for non-coplanar contact problems pervasive in whole-body manipulation. More recently, several approaches that consider the centroidal dynamics have been proposed to solve whole-body pattern generation problems, demonstrating the ability to compute efficiently kino-dynamic motions relevant for whole-body multi-contact problems (Herzog et al. (2016b); Carpentier and Mansard (2018); Dai et al. (2014)).

\section{Instantaneous Control}

Whole-body manipulation often necessitates the control of several concurrent, potentially conflicting, tasks which begs for approaches leveraging redundancy to optimally achieve all tasks whenever possible (see Del-Prete (2019) for more details). When certain tasks cannot be executed jointly, it is also often desirable to define priorities between tasks to ensure that high priority ones get executed first. For example, a robot walking towards an object to reach it might, in face of an unexpected disturbance, prioritize remaining balanced instead of aiming to reach the object at all costs.

Whole-body control approaches, stemming from operational space control methods extended to floating-base robots (Sentis and Khatib (2005); Henze et al. (2017)) have recently gained popularity. They enable the control of a set of tasks, typically written as desired closed-loop behaviors in task space and can also enforce some level of prioritization between tasks. Modern formulations of such controllers also enable the inclusion of inequality constraints and are typically formulated as the solution of an optimization problem (see for example Escande et al. (2014); De Lasa et al. (2010)). When tasks are linear in the decision variables (typically acceleration, actuation torques and contact forces), because the dynamic constraint imposed by the centroidal dynamics is also linear in these variables, the problem can be formulated as a quadratic program and solved sufficiently quickly to be used in fast 
control loops as in Herzog et al. (2016a). The explicit inclusion of tasks that enable impedance or admittance regulation before and after contacts (see example Dietrich et al. (2012)) or the regulation of interaction forces is another important aspect of such controllers. Optimal force distribution between contact points, for example, has been demonstrated to significantly improve the capabilities of the robots as in Righetti et al. (2013).

However, these formulations do not allow the inclusion of the predicted future states of the robot, potentially limiting the ability of the controllers to foresee potential problems and get trapped in local minima. Model predictive control approaches are therefore sought as a way to bridge the gap between motion planning and instantaneous control algorithms.

\section{Model Predictive Control}

Model predictive control has been proposed to generate complex motion over a short time horizon (typically less than a few seconds). For example, in Erez et al. (2013) it was used in simulation to allow a humanoid robot to open a valve, remove debris or drill a hole. In this interval the full robot dynamics is taken into account, including joint and torque limits or collision. The problem is formulated as an optimal control problem, where the free variables are the control vector for indirect formulations or both states and control for collocation. During the considered time horizon, contacts can be made or removed, significantly increasing the complexity of the problem. Indeed, the resulting optimal control formulations will then include complementarity constraints or will mix discrete (contacts) and continuous (motor control) variables. Note that in Erez et al. (2013) discrete variables are relaxed to be continuous. The resulting optimization problems are nonlinear and usually quite large. For a robot of 30 DOFs with a control period of $200 \mathrm{~Hz}$, the size of the problem quickly reaches over 9000 variables with only a $1.6 s$ time horizon. Model predictive control is routinely used for the control of legged locomotion with linear models of the center of mass following the seminal work of Wieber (2006b) and has recently been demonstrated on quadruped platforms with a full dynamics models as in Neunert et al. (2018). While numerous approaches are now being explored for an efficient resolution of the problem in the context of whole-body manipulation, including relaxation of the optimal control problem, warm-start or clever numerical implementation, the use of receding horizon control in fast control loops remains to be demonstrated for whole-body manipulation tasks on real robots.

\section{Motion planning}

In the case of whole-body manipulation, motion planning solves the following problem: is there a sequence of contacts involving the robot, the environment and the 
object in order to bring the object from a given starting state to a final state without collision. The set of motions that can be performed on the object are often fixed: sliding, pushing, rotating or lifting. The robot has also a limited number of its body parts that can be in contact with the object: grippers, wrists, forearms, chest, back, fingertips. The goal of motion planning is to find a sequence of contacts which is feasible for both the object and the robot. We can therefore explore both spaces simultaneously (Bouyarmane and Kheddar (2012)) or separately (Murooka et al. (2017)). A contact is validated once a posture at equilibrium is found for both the object and the robot. This equilibrium can be either quasi-static or dynamical.

A key ingredient is to find transitions between two possible contacts. Ideally it would be necessary to check all the physical constraints between the two contacts and all the possible trajectories. However, this is computationally too expensive to do in realistic scenarios. A typical strategy is to find a quasi-static motion connecting two contacts for which quasi-static postures of the robot exist. While it is a conservative approach, it significantly simplifies the problem. Dynamically balanced trajectories, which are faster, can be optimized in a second stage. However, this approach might fail when dynamically balanced contact transitions are necessary. See Fernbach et al. (2020) for a recent work in planning multi-contacts motion in an efficient way, while being dynamically balanced. The approach is still conservative in comparison to the full set of dynamically balanced trajectories.

Another manner to break the complexity of the problem is to use prior knowledge of the object and the robot. Affordance is playing a central role in whole-body manipulation. It is defining the actions that an actor can realize on an object and its consequences. These actions can be given beforehand by a human operator, automatically discovered through learning/statistical exploration, guided through heuristic, or provided from the study of humans (Borràs et al. (2017)). The interested reader can find an overview on motion planning and instantaneous control in Ibanez et al. (2017)

\section{Examples of Applications}

\section{Technological Level Readiness and field of applications}

Whole-body manipulation is not yet deployed in manufacturing. However several proof-of-concepts have been developed with various level of technical readiness. The main industrial interests are versatility and the potential to easily adapt to human environments. A versatile robot that can quickly be redeployed in various settings can potentially lower costs and the time necessary to adapt to new tasks. This is in stark contrast with current robotic solutions that are often very specialized. From that point of view, achieving whole-body manipulation with complex robots such as quadrupeds seems to be a promising market. 

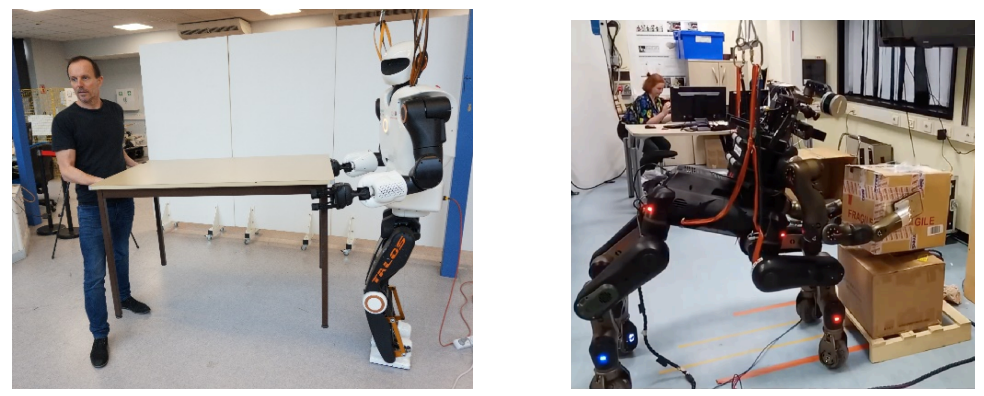

Fig. 2: The humanoid robot TALOS holding statically a table with a human in the COBOT project (left) and the quadruped Centauro moving a box with its hands Rolley-Parnell et al. (2018) (right).

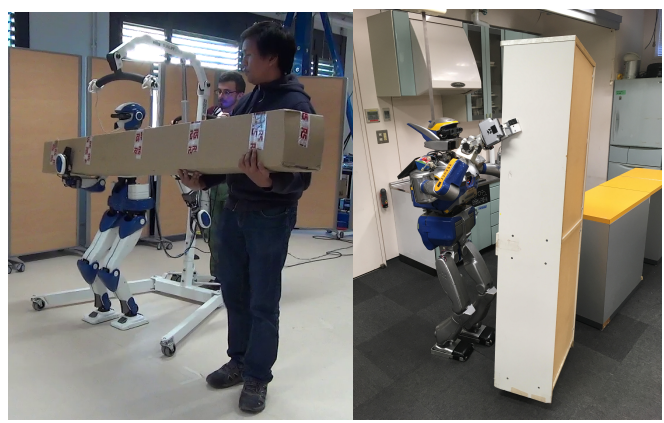

Fig. 3: (left) HRP-4 holding a large box with a human while walking (Agravante et al. (2019)) (right) HRP-2 pivoting a furniture (Murooka et al. (2017)).

\section{Humanoid robots}

The work presented in Murooka et al. (2015) is a clear demonstration of what can be achieved with whole-body manipulation. It described several postures that a HRP-2 humanoid robot takes to push heavy furniture. In contrast to traditional manipulation the robot uses surface to surface contact such as its back or its forearms to push furniture. In this case, the robot is able to provide up to $200 \mathrm{~N}$ of pushing force. The posture is subject to balance constraints that consider the whole system and great care must be taken with Coulomb friction. Indeed, when the tangential forces are reaching the friction coefficient limit, the system starts moving. More recently whole-body manipulation for carrying loads with a HRP-4 humanoid robot was also demonstrated (Agravante et al. (2019)). This approach decouples the centroidal dynamics for walking and instantaneous control. It allows the robot to carry boxes of $1.5 \mathrm{Kg}$ and buckets of $0.8 \mathrm{Kg}$. The DARPA Robotics Challenge (DRC) was an important international competition which demonstrated the current capabilities and 
limitations of robots performing locomotion and whole-body manipulation tasks, such as opening a door, shutting a valve or cutting out a dry wall. In particular, it showed that whole-body manipulation remains an open and difficult problem. Note that the humanoid robot winner of the DRC 2015 was equipped with wheels (Jung et al. (2019)). The interested reader can find more information on the results of this challenge in Spenko et al. (2017).

\section{Mobile manipulator}

Examples of whole-body manipulation mixing balance criteria and redundancy have also been shown on mobile platforms. Impressive fast whole-body manipulation has been demonstrated in Birbach et al. (2011) with the Justin robot. Balance constraints are less relevant as the mobile base has a large contact support on the ground. But an accurate geometrical calibration is necessary to be able to catch a flying ball before it touches the floor. In Kolhe et al. (2010), a Segway like structure pushes table and boxes. Boston Dynamics is also demonstrating possible application of their Handle robots (again a Segway like structure) in the context of logistics (Boston-Dynamics (2019a)). The robot is able to pick boxes from a pallet and to bring it to either another pallet, or to put it on automatic handling line. The robot speed is quite impressive. Note however that a special gripper is needed as well as a careful mechanical design such that the system can maintain balance despite its heavy load.

\section{Multi-legged robot}

Recent works show how quadrupeds can be used to manipulate objects. For instance several Spot minis were used to pull a truck (Boston-Dynamics (2019b)). The technology used in this video is based on brushless electric motors with low gear reduction ratio. Similarly, a new version of the HyQ robot from the Italian Institute of Technology, which is hydraulically actuated, is pulling a plane (Dynamic-LeggedSystems-Lab (2019)). In both cases the main problem is to have enough actuation power, few mechanical constraints and a balance system able to handle strong loads (in the order of several hundreds or thousands of Newtons). Centaur robots (RolleyParnell et al. (2018)), which consist of a quadruped lower body and a humanoid upper body, have also recently been developed (see Fig.2). This increases significantly the robustness of the robot with respect to balance (Rehman et al. (2016)). They can also be equipped with wheels to expand their mobility (Kamedula et al. (2018)). For instance the Momaro robot was very efficient during the DRC with this structure (Klamt and Behnke (2017)). 


\section{Future Directions for Research}

The routine deployment of whole-body manipulation strategies on real robots remains an open problem and fosters fundamental research questions. The design of robotic platforms capable of whole-body manipulation behaviors while remaining safe to operate in human environments is a central challenge that is tightly coupled to the control strategies deployed. Softer materials, denser contact sensing through artificial skins and higher density actuators constitute promising avenues of research. From the control and planning point of view, computational complexity remains a core limitation to provide real-time capable behavior generation and adaptation. It is very important to ensure sufficient reactivity in face of unexpected events or unmodeled dynamics with the environment. Promising research directions include efficient warm-start of the numerical optimization, principled relaxations of the optimization problem and a more systematic incorporation of multi-modal sensing, in particular visual perception and force/tactile feedback, in both control and planning loops. While machine learning techniques are seldom used for whole-body manipulation problems, we expect that they will play an important and complementary role to existing approaches. They can provide more accurate data-driven models of robot-environment interactions or the means to store and compress previous movements to be reused to warm-start online optimization. Scaling such techniques to high-dimensional state-action space and ensuring predictable safe operations will certainly constitute fundamental challenges for the application of machine learning. A major standing issue pertaining to all aspects of whole-body manipulation is ensuring that such behaviors will be robust to environmental uncertainty with safety guarantees when deployed in human environments.

\section{References}

Agravante DJ, Cherubini A, Sherikov A, Wieber PB, Kheddar A (2019) Human-humanoid collaborative carrying. IEEE Transactions on Robotics

Albu-Schäffer A, Ott C, Hirzinger G (2007) A unified passivity-based control framework for position, torque and impedance control of flexible joint robots. The international journal of robotics research 26:23-39

Birbach O, Frese U, Bäuml B (2011) Realtime perception for catching a flying ball with a mobile humanoid. In: IEEE/RAS , Int. Conf. Robotics and Automatio (ICRA)

Borràs J, Mandery C, Asfour T (2017) A whole-body support pose taxonomy for multi-contact humanoid robot motions. Science Robotics 2(13)

Boston-Dynamics (2019a) Handle robot reimagined for logistics. URL https://www.youtube.com/watch?v=5iV_hB08Uns

Boston-Dynamics (2019b) Spotmini robots pulling truck tests. URL https://www.youtube.com/watch?v=PBukEnjTJfc

Bouyarmane K, Kheddar A (2012) Humanoid robot locomotion and manipulation step planning. Advanced Robotics 26:10991126

Carpentier J, Mansard N (2018) Multicontact locomotion of legged robots. IEEE Transactions on Robotics 34(6):1441-1460 
Dai H, Valenzuela A, Tedrake R (2014) Whole-body motion planning with centroidal dynamics and full kinematics. In: 2014 IEEE-RAS International Conference on Humanoid Robots, IEEE, pp 295-302

De Lasa M, Mordatch I, Hertzmann A (2010) Feature-based locomotion controllers. In: ACM Transactions on Graphics (TOG), ACM, vol 29, p 131

Del-Prete A (2019) Task-Level Description, Springer

Dietrich A, Wimbock T, Albu-Schaffer A, Hirzinger G (2012) Reactive whole-body control: Dynamic mobile manipulation using a large number of actuated degrees of freedom. IEEE Robotics \& Automation Magazine 19(2):20-33

Dynamic-Legged-Systems-Lab (2019) Hyqreal robot release: Walking robot pulls a plane (extended version). URL https://www.youtube.com/watch? $\mathrm{v}=\mathrm{pLsNs} 1 \mathrm{ZS} \_\mathrm{TI}$

Englsberger J, Werner A, Ott C, Henze B, Roa MA, Garofalo G, Burger R, Beyer A, Eiberger O, Schmid K, Albu-Schlaffer A (2014) Overview of the torque-controlled humanoid robot toro. In: IEEE/RAS Int. Conf. on Humanoid Robotics

Erez T, Lowrey K, Tassa Y, Kumar V, Kolev S, Todorov E (2013) An integrated system for real-time model predictive control of humanoid robots. In: IEEE-RAS Int. Conf. on Humanoid Robots, IEEE, pp 292-299

Escande A, Mansard N, Wieber PB (2014) Hierarchical quadratic programming: Fast online humanoid-robot motion generation. The International Journal of Robotics Research 33(7):10061028

Fernbach P, Tonneau S, Stasse O, Carpentier J, Taïx M (2020) C-croc: Continuous and convex resolution of centroidal dynamic trajectories for legged robots in multi-contact scenarios. IEEE Transactions on Robotics Accepted

Hasegawa S, Wada K, Okada K, Inaba M (2018) Detecting and picking of folded objects with a multiple sensor integrated robot hand. In: IEEE/RSJ International Conference on Intelligent Robots and Systems, IROS, pp 1138-1145

Henze B, Dietrich A, Roa MA, Ott C (2017) Multi-contact balancing of humanoid robots in confined spaces: Utilizing knee contacts. In: 2017 IEEE/RSJ International Conference on Intelligent Robots and Systems (IROS), pp 697-704

Herzog A, Rotella N, Mason S, Grimminger F, Schaal S, Righetti L (2016a) Momentum control with hierarchical inverse dynamics on a torque-controlled humanoid. Autonomous Robots 40(3):473491

Herzog A, Schaal S, Righetti L (2016b) Structured contact force optimization for kino-dynamic motion generation. In: 2016 IEEE/RSJ International Conference on Intelligent Robots and Systems (IROS), IEEE, pp 2703-2710

Ibanez A, Bidaud P, Padois V (2017) Humanoid Robots: A Reference, Springer, chap OptimizationBased Control Approaches to Humanoid Balancing

Jung T, Lim J, Bae H, Oh J (2019) Mechanism Design Outline of Hubo, Springer Netherlands, pp 615-635

Kajita S, Kanehiro F, Kaneko K, Yokoi K, Hirukawa H (2001) The 3d linear inverted pendulum mode: A simple modeling for a biped walking pattern generation. In: Proceedings 2001 IEEE/RSJ International Conference on Intelligent Robots and Systems. Expanding the Societal Role of Robotics in the the Next Millennium (Cat. No. 01CH37180), IEEE, vol 1, pp 239-246

Kamedula M, Kashiri N, Tsagarakis NG (2018) On the kinematics of wheeled motion control of a hybrid wheeled-legged centauro robot. In: IEEE/RSJ Int. Conf. on Intelligent Robots and Systems (IROS)

Kendall A, Grimes M, Cipolla R (2015) Posenet: A convolutional network for real-time 6-dof camera relocalization. In: The IEEE International Conference on Computer Vision (ICCV), pp 2938-2946

Klamt T, Behnke S (2017) Anytime hybrid driving-stepping locomotion planning. In: IEEE/RSJ Int. Conf. on Intelligent Robots and Systems (IROS)

Kolhe P, Dantam N, Stilman M (2010) Dynamic pushing strategies for dynamically stable mobile manipulators. In: IEEE/RAS , Int. Conf. Robotics and Automatio (ICRA) 
Lee MA, Zhu Y, Srinivasan K, Shah P, Savarese S, Fei-Fei L, Garg A, Bohg J (2019) Making sense of vision and touch: Self-supervised learning of multimodal representations for contactrich tasks. In: 2019 International Conference on Robotics and Automation (ICRA), IEEE, pp 8943-8950

Mesesan G, Englsberger J, Garofalo G, Ott C, Albu-Schäffer A (2019) Dynamic walking on compliant and uneven terrain using dcm and passivity-based whole-body control. In: The IEEE International Conference on Humanoid Robots (ICHR)

Mittendorfer P, Yoshida E, Cheng G (2015) Realizing whole-body tactile interactions with a selforganizing, multi-modal artificial skin on a humanoid robot. Advanced Robotics

Murooka M, Nozawa S, Kakiuchi Y, Okada K, Inaba M (2015) Whole-body pushing manipulation with contact posture planning of large and heavy object for humanoid robot. In: IEEE/RAS , Int. Conf. Robotics and Automatio (ICRA)

Murooka M, Ueda R, Nozawa S, Kakiuchi Y, Okada K, Inaba M (2017) Global planning of wholebody manipulation by humanoid robot based on transition graph of object motion and contact switching. Advanced Robotics 31(6):322-340

Neunert M, Stäuble M, Giftthaler M, Bellicoso CD, Carius J, Gehring C, Hutter M, Buchli J (2018) Whole-body nonlinear model predictive control through contacts for quadrupeds. IEEE Robotics and Automation Letters 3(3):1458-1465

Nori F, Traversaro S, Fallon M (2018) Sensor Fusion and State Estimation of the Robot, pp 1-29

Orin DE, Goswami A, Lee SH (2013) Centroidal dynamics of a humanoid robot. Autonomous Robots 35(2-3):161-176

Pavlichenko D, Rodriguez D, Schwarz M, Lenz C, Periyasamy AS, Behnke S (2018) Autonomous dual-arm manipulation of familiar objects. In: IEEE-RAS Int. Conf. on Humanoid Robots

Rehman BU, Focchi M, Frigerio M, Goldsmith J, Caldwell DG, Semini C (2016) Design of a hydraulically actuated arm for a quadruped robot. In: CLAWAR

Righetti L, Buchli J, Mistry M, Kalakrishnan M, Schaal S (2013) Optimal distribution of contact forces with inverse-dynamics control. The International Journal of Robotics Research 32(3):280-298

Rioux A, Suleiman W (2018) Autonomous slam based humanoid navigation in a cluttered environment while transporting a heavy load. Robotics and Autonomous Systems pp 50-62

Rolley-Parnell E, Kanoulas D, Laurenzi A, Delhaisse B, Rozo L, Caldwell DG, Tsagarakis NG (2018) Bi-manual articulated robot teleoperation using an external rgb-d range sensor. In: 2018 15th International Conference on Control, Automation, Robotics and Vision (ICARCV), pp 298-304, DOI 10.1109/ICARCV.2018.8581174

Sentis L, Khatib O (2005) Synthesis of whole-body behaviors through hierarchical control of behavioral primitives. International Journal of Humanoid Robotics 2(04):505-518

Spenko M, Buerger S, Iagnemma K (2017) Special issue:the darpa robotics challenge finals

Stasse O, Flayols T (2019) Springer, chap An overview of humanoid robots technologies

Wensing PM, Wang A, Seok S, Otten D, Lang J, Kim S (2017) Proprioceptive actuator design in the mit cheetah: Impact mitigation and high-bandwidth physical interaction for dynamic legged robots. IEEE Transactions on Robotics 33(3):509-522

Wieber PB (2006a) Holonomy and nonholonomy in the dynamics of articulated motion. In: Fast motions in biomechanics and robotics, Springer, pp 411-425

Wieber PB (2006b) Trajectory free linear model predictive control for stable walking in the presence of strong perturbations. In: 2006 6th IEEE-RAS International Conference on Humanoid Robots, IEEE, pp 137-142

\section{Cross-references}

A. Del-Prete, Task-Level Description, Enclycopedia of Robotics, 2019, Springer 


\section{Acknowledgements}

We would like to thanks the authors of Agravante et al. (2019) Rolley-Parnell et al. (2018) and Murooka et al. (2017) for providing the images in Fig.2 and Fig.3. 